\title{
Section introduction \\ Who would have thought it! Influence on outcome of radiotherapy, Ki67 and stroma. Introduction to Session 5
}

Alan Rodger

Retired Medical Director, Beatson West of Scotland Cancer Centre, Great Western Road, Glasgow, G12 0YN, Scotland, UK

Corresponding author: Alan Rodger, alanrodger@f2s.com

Published: 18 December 2009

This article is online at

http://breast-cancer-research.com/supplements/11/S3/S13

In breast cancer there are clearly a number of factors that counter-intuitively influence outcome or for which there is a change, after a considerable time, in longstanding evidence that outcome was not affected. The latter is well represented by the effect of postoperative radiotherapy in early breast cancer on overall survival. There was clear evidence that such treatment reduced local recurrence risk (by about two-thirds) and risk of death from breast cancer (by one-sixth [1]), but until 1997 evidence suggested treatment would not improve overall survival. The results in that year of two randomised trials in high-risk patients showed that the addition of postmastectomy radiotherapy to adjuvant chemotherapy improved overall survival $[2,3]$.

Ragaz argues from the updated results of these pivotal Canadian and Danish trails, from survival trends observed in British Columbia, Canada and from the data presented consistently from 1990 to 2005 by the Early Breast Cancer Trialists' Collaborative Group that this effect on outcome is real [4]. The reality is explained by the record reduction over this period in post-radiotherapy acute and late morbidity due to improved techniques and dosimetry.

Ragaz also indicates that, due to lack of radiotherapy trials, there is no clarity on what needs to be irradiated, while the question of who needs to be irradiated can no longer be answered simplistically on the basis of the number of nodes involved. The histopathological extent of nodal involvement judged by the percentage of involved nodes, by the spread within a node and by the presence of extracapsular spread all indicators of risk of systemic relapse - is more critical than the indicators of increased risk of local failure.

The need for more trials in radiotherapy is underlined by the fact that this paradigm shift in attitude and assessment of benefit occurred when the results of the pivotal randomised trials were published in 1997 showing that postoperative radiotherapy could improve long-term overall survival and not just cause long-term morbidity.
Breast Cancer Research 2009, 11(Suppl 3):S13

(doi:10.1186/bcr2432)

(c) 2009 BioMed Central Ltd

That a single measurement of Ki67 does predict long-term outcome is discussed by Dowsett and colleagues [5], but with the caveat that such a single assay may not deliver all of the required information. For prognosis alone, a single pretreatment assay suffices - but for predictive information, a second post-treatment assay in the neoadjuvant case is needed. Further assays offer even more information for drug development studies or of drug resistance mechanisms.

While breast stroma has been of passing interest to pathologists since at least the 1960s, when elastosis was considered a possible marker of prognosis, the hypothesis that malignant epithelial cells alone are prognostic and the supporting (stromal) tissues are of no prognostic influence has generally been the focus of research and clinical interest. This no longer stands up to scrutiny. Howell and colleagues describe well how stroma is recruited by tumours, is variable from tumour to tumour, and how tumour genes may be downregulated (for adhesion) or upregulated (facilitating cell movement) [6] - all potentially affecting outcome. Stroma does not, however, integrate with other prognostic factors.

Stroma is already targeted in various ways in clinical situations. Bevacizumab and the bisphosphonates are already in regular and effective use. There is, therefore, opportunity for further improvement in outcomes as other stromal targets are explored.

'Who would have thought it!' is a statement and not a question. Many did think it, and that is why the critically important trials in postmastectomy radiotherapy were carried out even though some trialists expected the old paradigm to be confirmed. Newer technologies now enable pathologists and clinicians to revisit hypotheses on stroma mooted 40 years ago. Neoadjuvant treatments also facilitate assessment of the prognostic effects of molecular and genetic factors. Clearly, it is having the thought that counts.

\section{Competing interests}

The author declares that they have no competing interests. 


\section{Acknowledgement}

This article has been published as part of Breast Cancer Research Volume 11 Suppl 3 2009: Controversies in Breast Cancer 2009. The full contents of the supplement are available online at http://breastcancer-research.com/supplements/11/S3.

\section{References}

1. Early Breast Cancer Trialists' Collaborative Group: Favourable and unfavourable effects on long-term survival of radiotherapy for early breast cancer: an overview of the randomised trials. Lancet 2000, 355:1757-1770.

2. Ragaz J, Jackson SM, Le N, Plenderleith IH, Spinelli JJ, Basco VE, Wilson KS, Knowling MA, Coppin CML, Paradis M, Coldman AJ, Olivotto IA: Adjuvant radiotherapy and chemotherapy in nodepositive premenopausal women with breast cancer. $N$ Engl J Med 1997, 337:956-962.

3. Overgaard M, Hansen PS, Overgaard J, Rose C, Andersson M, Bach F, Kjaer M, Gadeberg CC, Mouridsen HT, Jensen M-B, Zedeler K for the Danish Breast Cancer Cooperative Group 82b Trial: Postoperative radiotherapy in high-risk premenopausal women with breast cancer who receive adjuvant chemotherapy. N Engl J Med 1997, 337:949-955.

4. Ragaz: Breast Cancer Res 2009, 11(Suppl 3):S14.

5. Dowsett M, A'Hern R, Salter J, Zabaglo L, Smith I: Who would have thought a single Ki67 measurement would predict longterm outcome? Breast Cancer Res 2009, 11(Suppl 3):S15.

6. Howell $A$, Landberg $G$, Bergh J: Breast tumour stroma is a prognostic indicator and target for therapy. Breast Cancer Res 2009, 11(Suppl 3):S16. 